\section{ARC182: Drafting and Drawing}

\author{
JONATHAN LOUIE
}

Syracuse University the architects' objective is given from the outside. ${ }^{7}$ Unlike the autonomy of other artistic practices architects depend on clients, builders, and patrons to execute their work. To Allen it is a matter of images, that the architect is reliant on modes of textual and visual communication to construct their ideas.

To think in images, as Vilhelm Flusser suggests, makes our internal imagination communicable to others; and the construction of Pictures are a means of fixing the products of this 'imaginative withdrawal.' In his book, The Future of Writing, Flusser proposes that writing does not have a future. 'That everything that is currently conveyed in writing-and much that cannot be-can be recorded and transmitted by other means. ${ }^{18}$ While previous overlaps of imagery from art to architecture focused on the translation of perspectival space, and later the disruption of space sthrough the fractured cubist plane. To be straightforward and to just think in images whether they are Construction Drawings, As-Built Drawings, or EndgameDrawings - alters traditional habits of communicating and interpreting between the mind and the hand, thinking and imaging. in architectural design investigation, development, and presentation. Exposure to CAD (Computer Aided Design)." While at first glance one might assume that the course teaches proper means and methods for drafting or drawing architectural ideas in CAD. And introduces students to the language of codes and symbols that communicate a design through drawing. Or the process of drawing production from design concept to drafting production. But upon second thought, I think there is more to say. After all, in an increasingly image saturated world where images operate transitively, ${ }^{4}$ and their abundance are altering our habits of communication and interpretation. ${ }^{5}$ There is an increasingly blurry distinction between the codification of drafting and drawing in architecture offices today. Begging the question, if architectures means and ends rely on imaging, how can architectural ideas develop from mixing meaning in its' types of images?

It is important to note that the subtle linguistic swap from drafting to drawing, vastly alters the conception of the 'Introduction to Representation II' course, from drafting as communication (allographic art) ${ }^{6}$ to a celebration of drawings effects and autonomous qualities. From a broader point of view, the line between drafting and drawing distinguishes: the rationality of an architectural practice from the creative and intellectual explorations of an architectural project. In the opening paragraph of 'Practice vs Project,' Stan Allen said it best: 'After all,

\section{CONSTRUCTION DRAWINGS}

To distinguish Construction Drawing (also known as Record Drawings) from Endgame Drawing I'd like to briefly return to Vilhelm Flussers' ideas concerning the image and the text; the regular stuff that architects deploy to alter our habits of communication and interpretation with architects, consultants, and builders in the building industry. In a world increasingly saturated by new media, Flusser points to Techno-Images ${ }^{9}$ - photography, televisuality, and digitality - that transcode information from the textual world as images. They take the place of the linear display of text and the binary system of computation, by depicting an open surface with multiple interpretations. While Flussers' work primarily highlights the interchange between text and 20th century media technologies, his ideas offer a unique way into understanding the productive exchange between endgame drawing and drafting in the work of young architects and students in the post-digital age.

Unlike Endgame Drawings, which are an 'open surface' for interpretation, Construction Drawings convert text to a drawing system that is not meant for misinterpretation or misrepresentation. While drafting and drawing have a few things in common: both begin with a mark, both are an assembly of points and lines, and, both are an act of representing a 
subject. Over the years, Drafters have established representational conventions made up of standard drawing orientations (projection), line types, scales, units of measurement, symbols, and notations that constitute a visual language for communication. Contractually, Construction Drawings house information that binds an agreement between client and contractor, as well as, between architect and client. And the interpretation of its' content determines both pricing and assembly. Simply put, Drafting is a representational system that communicates how something is or is to be constructed in the future; and the compilation of documents into a Construction Drawing Set establishes a framework for a buildings construction to come.
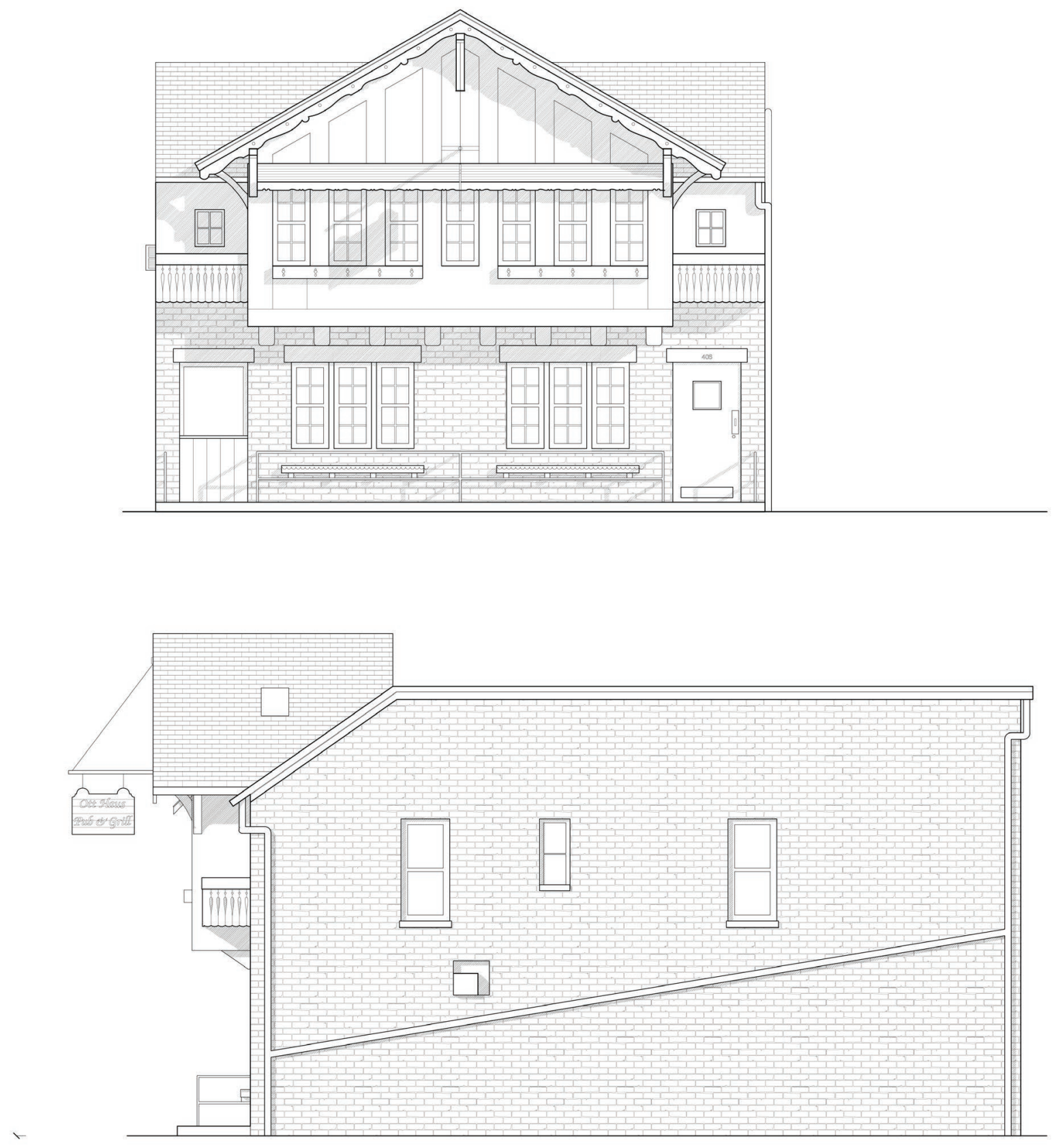

Figure 1. Front and Side Elevations of Haus Ott, Swissness Applied, Weiqiao Lin, Professor Nicole McIntosh. 


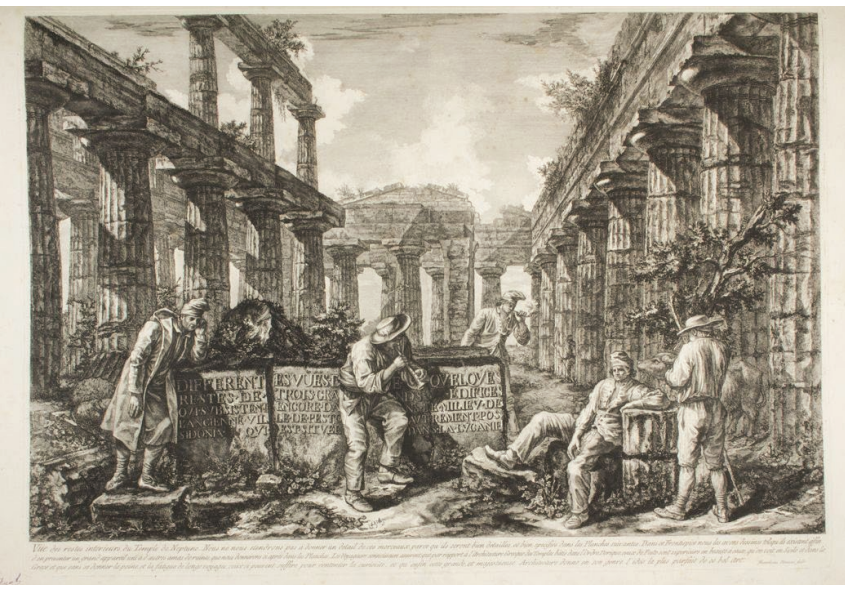

Figure 2. Temple of Neptune, looking through the peristyle from the north-west corner, showing the internal colonnades and the Basilica in the distance, Giovanni Battista Piranesi.

\section{AS-BUILT DRAWINGS}

Despite their familiar appearance, they share notations, symbols, and abbreviations, with other technical rawings. As-Built Drawing Sets are a revised set of drawings by the contractor that documents an existing condition upon completion of a project. They record the realities of the construction, including any design changes made in the field: change orders, component relocations, and rerouting of distribution systems. They describe a present state and serve as a record for future system changes and additions.

\section{ENDGAME DRAWING}

While for centuries architects have worked to develop drafting techniques for communicating their designs, foregrounding the act of drafting as a genesis ${ }^{10}$ of the discipline, it is equally important to acknowledge the drawing as an act of architecture itself. In 'Perfect Acts of Architecture,' Jeffrey Kipnis defines the Endgame Drawing as an architectural act, he notes that it must function in three ways: as an innovative design tool, as the articulation of a new direction, or as a creation of consummate artist merit. ${ }^{11}$ To Kipnis, for a drawing to meet all three demands is a Perfect Act of Architecture; one that is not influenced by clients nor consultants and considers the conception and articulation of a project, how it begins and ends, through the drawing.

Endgame Drawings are exemplars of an architects predisposition to 'think in images.' It beholds all the ideas of the architect minus the constraints of the client, the imperfections of the builder, or the influence of the consultants. The drawing relies on the limitations of its' medium - the flatness of the page, edge of the paper, and the technique - to represent the matter at hand. Drawing constraints, which are not typically significant for the act of building construction, are significant as the means for developing an architectural thought. For generations architects deployed the Endgame Drawing as an apparatus that simultaneously is worked on and communicates an architectural project as a pursuit for an internal logic. They are an act of architecture.

\section{AS-FOUND DRAWINGS PT. 1}

Perhaps an early predecessor for the collapse of As-Built and Endgame Drawings can be seen in Giovanni Battista Piranesis' documentation of the Temples at Paestum (1777). They are drawings of an archeological site, along the Gulf of Naples, that record three Doric Temples - the Basilica, the Temple of Neptune, and the Temple of Ceres - as built characters in a present state of decay.

While As-Built drawings are typically prepared by contractors as documentation of the present state of a building assembly. The As-Found, to Alison and Peter Smithson, are 'not only adjacent buildings but all those marks that constitute remembrancer in a place that are to be read...'This includes the state of the scene that make it specific-to-a-place. The meticulous perspectival documentation of Paestum captures the temple setting in an as-found condition, in various states of disarray, as the architectural backdrop for eighteenth century loiterers, traveling merchants, and shepherds that occupy the rustic setting. The drawings collapse the appearance of two cultural periods (mid-6th century BC and mid-18th century) in a single image by acknowledging all the 'prosaic things' of the scene that show how it came to be. Within this drawing trajectory, Nicole Mclntoshs' Tell No Cabbage (Swissness Applied), with a transposition of elements from Swiss and American vernacular, finds its distant ancestor in Piranesi's drawings of Paestum.

Mclntoshs' Tell No Cabbage (Swissness Applied) is a series of As-Found drawings that depict eighteen buildings in the commercial district of New Glarus whose appearance is translated from select photographs of Swiss Chalets. For the past couple years Mclntosh and her students are documenting the present condition of a town that has been 'Swissifying' its' appearance since the $1950 \mathrm{~s}^{\prime}{ }^{12}$ Each drawing set, comprised of scenes with façade and side elevations, display the planned and ad-hoc assembly systems of a vernacular condition that are built up over time.

Like Piranesis' Temples at Paestum, or Clark Thenhaus' As-Built drawings of San Francisco Victorian vernacular, when looking at Mclntoshes' Tell No Cabbage (Swissness Applied) it is important to note its' multiple values embedded in its' appearance. For example, on one hand, each drawing in the series displays the misalignment of the Swiss image applied onto an American structure, calling attention to the mundane realities of everyday life in Wisconsins' Little Switzerland. On the other, the drawings reveal an alternative image to the traditional Swiss Chalet. The cannily crafted linework simultaneously portrays overturned trash cans, window additions, and ad hoc electrical conduit, that sit happily alongside built swiss motifs, such as: gable roofs with decorative look out beams, scherenschnitt balconies, and shutters that appropriate multiple cultural conditions. Resulting in strange and abnormal exterior qualities that are not Swiss or American but construct another cultural reality. 


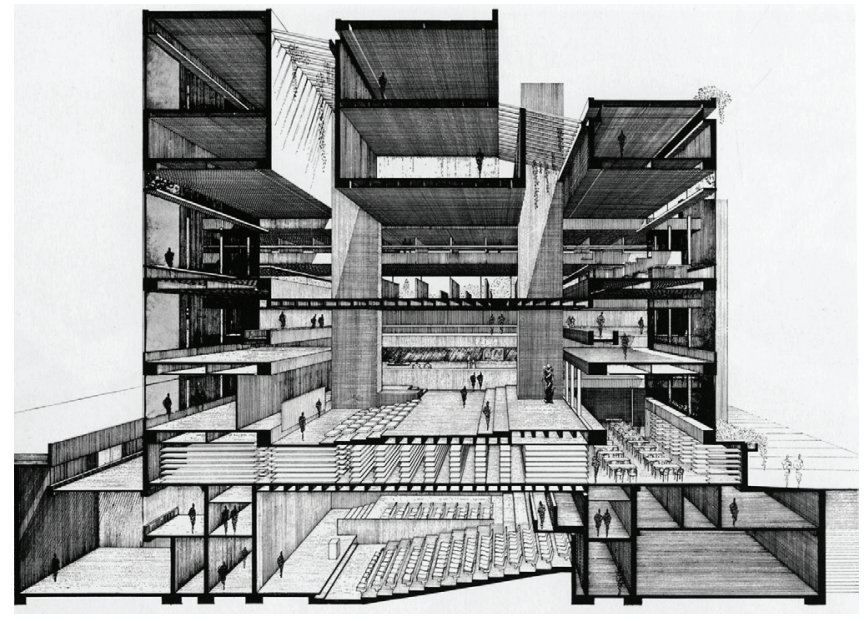

Figure 3. Sectional Perspective of the Yale Art and Architecture Building, Paul Rudolph.

Unlike a photograph that indiscriminately captures everything within its' frame, Tell No Cabbage (Swissness Applied) focuses the viewer on the local construction techniques and newly established details that reference the written descriptions and photographs inthe New Glarus Building Codes. It accepts the awkwardness in the clumsy details, misalignments of construction standards, and low fidelity motifs that are crafted by artisans from another time and place. The drawings highlights the value of the building codes as a basis for cultural appropriation by documenting an existing condition of image translation from image to surface assembly, one medium to another.

\section{AS-FOUND DRAWINGS PT. 2}

In his article, 'Rendering the Surface: Paul Rudolph's Art and Architecture Building at Yale,' Timothy Rohan recounts the story of certain Paul Rudolph drawings that never quite left the confines of his workplace. ${ }^{13}$ In lieu of drafting orthographic projections, Rudolph, who fancied his architecture office as more of a boutique atelier, would painstakingly construct the drawings by meticulously ruling in the characteristics of the projects surfaces. While for years the surface has been a plane for the translation between drawing and building. In his atelier the drafting board was both a plane for constructing drawings (and for the architect to imagine a buildings assembly), as well as, the plane for imaging the exterior surface of the building. Rudolphs sectional perspectives collapse the drawing and building surface as a means of displaying both a space and its' qualities. They were an interoffice design tool, a means for Rudolph to intellectually develop the character of a building by physically representing it through image.

According to Adrian Forty, the pen-and-ink rendering came from Rudolphs' avoidance of models during the development of the design, because: "The model cannot readily indicate details or materials, ${ }^{14}$ and that the drawn rendering better conveys the material substance of his buildings. For example, compare the section perspective drawings of Rudolphs' Yale Art and Architecture Building side by side with the rooms and surfaces they depict. The ruled markings on the drawings surface (with all of its graphite induced characteristics and effects) act as a material study for the concrete bush hammering to come. To Reyner Banham, it was one of the very few buildings that "... when photographed, was exactly like a drawing, with all the shading on the outside coming out as if it were ruled in with a very soft pencil."15 In Rudolphs' drawings, the lines and hatching depict the atmospheric qualities and surface characteristics of the bush hammered concrete. They work to visually soften the hard edges of the surface by repetitively marking its' substrate.

In this vein, Surfaces of Slocum, an assignment from an Arc 182 course taught by the author, set freshman students to the task of documenting and constructing an image of a material surface at full scale. Over the span of two weeks students photographed the rooms of Slocum Hall, then processed the photographs through multiple software (Illustrator and Photoshop) to capture the color, texture, and assembly of asfound surfaces through vector line and fill.

Surfaces of Slocumre configures the image data from the photograph into a new surface. Resulting in something that, according to Anne Friedberg, is a virtual approximation of reality: it appears to be, but is not, of the same materiality as what it represents. ${ }^{16}$ Connecting visuality with architecture by considering the surface substrate as a link between immaterial imagery and the presence of matter. At the end of the assignment students arrayed their prints throughout the school. Resulting in an impromptu exhibition of physical matter whose appearance strays from the original material to reveal the layers of software protocols that mediate a digital drawing today.

\section{NOT DRAWING OR DRAFTING, IMAGING}

By focusing on the medium of drawing as a frame for the articulation of an architectural thought. Arc 182 collapses Endgame drawing and drafting into As-Found Drawings, modes of imaging that acknowledge the 'prosaic things' around us by portraying the multivalent identities of drawings.

After four years of teaching 'Arc 182' and several days writing this paper. I'd like to end this article with one quick thought. In early pedagogical sequences around the country we formally teach students how to draft as a tool for communicating the complete execution of their intent. In a contemporary society saturated with imagery, if the value of a drafted drawing set is not only to convey the complete and correct information necessary for the execution of a building. How can misreading its identity lead to the development of architectural projects today? 


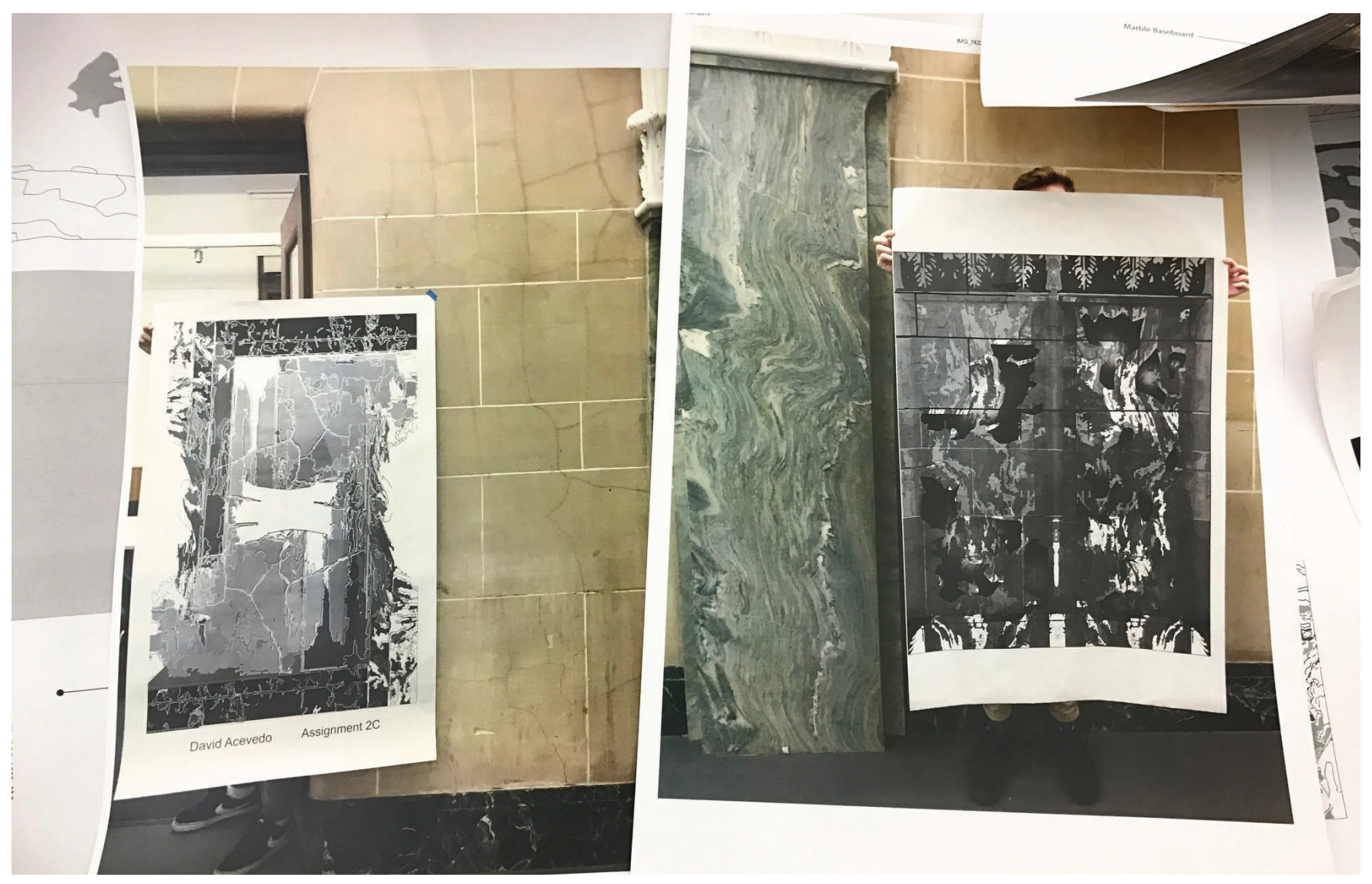

Figure 4. Photograph of Surfaces of Slocum Drawings by David Acevedo and Lawrence Sprague, Courtesy of Author.

\section{ENDNOTES}

1 Mark Linder, "Doing Imaging Things," Props Paper 27, accessed December 31, 2018. http://propspaper.com/issues/issue-27/issue-27.

2 This is the course number designation for the second representation course taught in Spring semester of the Foundation Year in an Undergraduate Education at Syracuse University.

3 This a course number designation for the first representation course taught in the Fall semester of the Foundation Year in an Undergraduate Education at Syracuse University.

4 Jacques Rancierre, "The Future of the Image," in The Future of the Image, trans., G. Elliot (London: Verso, 2007), 3.

5 Linder, "Doing Imaging Things."

6 In his quest to categorize artforms in Languages of Arts, Nelson Goodman attempts to classify artforms to merge the humanist and artistic approaches with the rest of the reality we live in (namely science and ordinary knowledge). To Goodman due to Technical Drawing, Architecture was an allographic art. Like musical compositions, it was a device constructed by the author but interpreted by another. Despite the instruction set staying the same, the result of the translation is out of the authors control. Resulting in two outputs never quite being the same. Nelson Goodman, Languages of Arts: An Approach to a Theory of Symbols, second edition (Indianapolis: Hackett Publishing Company, 1976).

7 Stan Allen, “Introduction: Practice vs Project," in Practice: Architecture, Technique, and Representation (London: Routledge, 2009), XIV.

8 Allen, XIV.

9 Vilhelm Flusser, Does Writing Have a Future? (Minneapolis: University of Minnesota Press, 1989)

10 Flusser, 67

11 Jeffrey Kipnis, "An Introduction to a Perfect Act," in Perfect Acts of Architecture (New York: The Museum of Modern Art, 2002), 12.

12 Nicole McIntosh, "Swissness Applied," presented at the ACSA Fall 2018 Conference, Milwaukee, WI, October 12, 2018.

13 Timothy Rohan, "Rendering the Surface: Paul Rudolph's Art and Architecture Building at Yale," Grey Room 1 (2000): 84-107.
14 Adrian Forty, Concrete and Culture: A Material History (London: Reaktion Books, 2013).

15 Reynher Banham, "Convenient Benches and Handy Hooks: Functional Considerations in the Criticism of the Art of Architecture," in The History, Theory and Criticism of Architecture: Papers from the 1964 AIA-ACSA Teacher Seminar, ed., Marcus Whiffen (Cambridge, MA: The MIT Press): 102.

16 Anne Friedberg, "Introduction: The Virtual Window," in The Virtual Window (Boston: The MIT Press, 2009): 8. 